\title{
Comparative study of Pulsed IR Thermography and Ultrasonic Testing to obtain a porosity mapping of oxide/oxide CMC specimens
}

\author{
by L. Gavérina *, A.Debarre*, C. Trottier *, F. Passilly *and J-M. Roche *
}

*ONERA, Materials and Structures Department, Châtillon, 92320, France, ludovic.gaverina@onera.fr

\begin{abstract}
In this paper, a Non Destructive Testing approach by active infrared thermography is used to investigate porosity in oxide/oxide CMC specimens. In this study, an experimental bench is developed using pulsed thermography in reflection or transmission modes coupled with transient heat transfer modelling in order to detect inhomogeneities. Results obtained are discussed and compared to ultrasonic testing which is commonly used in Non Destructive Tests.
\end{abstract}

\section{Introduction}

The implementation of ceramic matrix composite (CMCs) in aircraft engines is constantly increasing. During the elaboration process of these materials, it is important to survey the micro-porosity distribution to check the homogeneity of porosity and the absence of large porosity in the specimen. A pore is defined as an inclusion of air and the porosity rate is defined as the volume of pores compared to the volume of the material. This latest value is directly related to mechanical performance of this specimen and so must be assessed. To validate the porosity rate many experimental techniques can be used: destructive ones (as Scanning Electron Microscopy) or non-destructive ones (as ultrasonic testing, X-ray tomography ...). These techniques make it possible to obtain a porosity map to analyze the homogeneity of porosity in the specimen. In this work, we propose to compare two non-destructive characterization methods to investigate the porosity distribution. The first one is a pulsed IR thermography, which is a conventional non-destructive testing approach. It offers many advantages, such as rapid measurement and simple set-up. The easy physical interpretation enables a correlation between the temperature distribution and the porosity distribution to obtain a mapping of porosity and to estimate the porosity rate. The second technique is ultrasonic testing. This method is conventional too and well known for this kind of materials and so is used as reference technique for the results comparison.

\section{Experimental set-up}

For the investigation, two halogen lamps whit $6 \mathrm{~kJ}$ each are used to provide the thermal solicitation to the sample. The surface of the specimen is preliminary painted to have an emissivity close to 0.97 . The IR camera which was used during experiments is a FLIR X6540sc MWIR camera $(640 \times 512$ pixels, NETD $20 \mathrm{mK}$, pitch $15 \mu \mathrm{m}$, spectral band $1.5-5 \mu \mathrm{m})$. The maximum frame rate of the camera was $150 \mathrm{~Hz}$. The ultrasonic testing is performed in a water tank, in transmission configuration with two probes at $5 \mathrm{MHz}$ of central frequency.

Experiments in transmission and reflection mode were carried out on academic oxide/oxide CMC specimens, which incorporate porosities of different sizes and locations. The dimensions of the square oxide/oxide CMC sample, presented in Figure 1, are $160 \mathrm{~mm} \times 150 \mathrm{~mm} \times 2.3 \mathrm{~mm}$. The thermal properties of the material are the following: thermal conductivity $\lambda=3.49 \mathrm{~W} \cdot \mathrm{m}^{-1} \cdot \mathrm{K}^{-1}$, volumetric heat capacity $\rho C p=1.92^{*} 10^{-6} \mathrm{~J} \cdot \mathrm{m}^{-3} \cdot \mathrm{K}^{-1}$.

a)

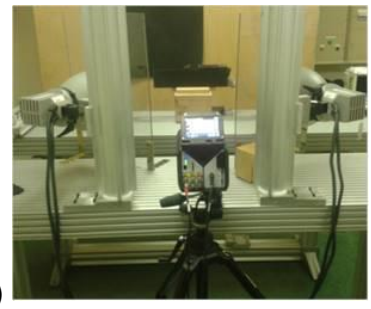

b)

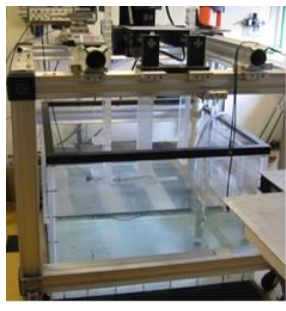

c)

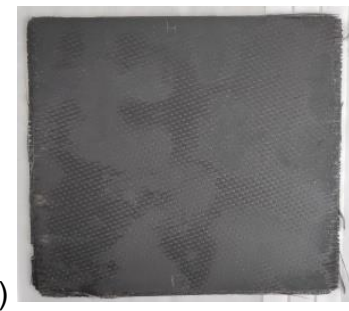

Fig. 1. (a) Photography of the thermography set-up, (b) the ultrasonic bench and (c) photography of the sample.

\section{Porosity map}

Figure.2.a shows the normalized thermogram of rear face of the oxide/oxide CMC specimen submitted to a flash heating. Regarding the thermograms in transmission mode, they are obtained by dividing the temperatures by the final asymptotic value. The healthy part is identified in blue color while the damaged one is in red color. The presence of 
the defects is clearly seen. Regarding the reflection mode, the Thermographic Signal Reconstruction (TSR) technique [1] is proposed to improve the detection of defect after the flash heating. In this case, a fitting of the logarithm of the temperature time-evolution is performed by a polynomial of degree 7 . In order to reveal the defects on the surface of the specimen among the porosity inside the specimen, the second order derivative [2] has been applied to TSR images. These results are shown in figure 2.b. In short time, the defects appear directly in the second order derivative image. It is means that the defect can be some porosities or lack of matter on the surface of the specimen.

a)

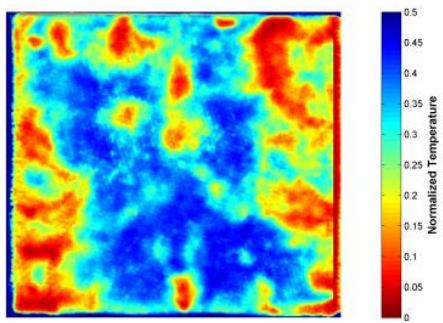

b)

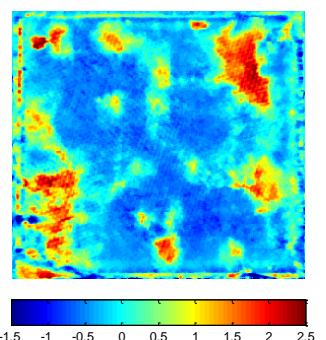

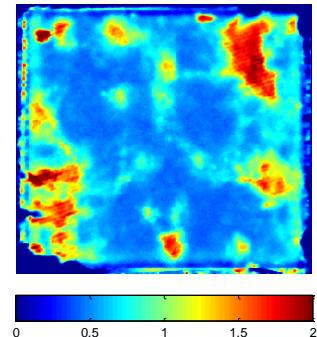

Fig. 2. (a) Rear-face thermograms at optimal time (0.19s); (b) front-face $2^{\text {nd }}$ logarithmic TSR thermograms at early time $(0,05 \mathrm{~s})$ (left) and late time (2s) (right).

A quantitative analysis has been done: figure 3.a shows a thermal diffusivity mapping obtained with the Degiovanni's method [3] by analysing the pulsed response of the rear-face of the specimen. The thickness of the specimen is not constant, therefore to improve the accuracy of the estimation for each pixel of infrared image, the thermal diffusivity has been corrected (figure 3.b) based on a thickness mapping (figure 3.c) of the sample (64 values of thickness).

\section{a)}

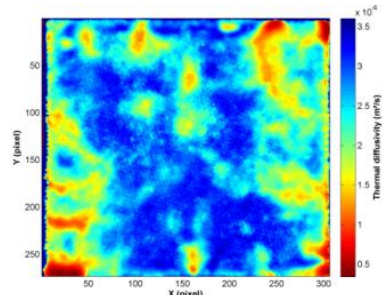

Fig. 3. Mapping of the thermal diffusivity: of the specimen, (b) thermal diffusivities estimated from a mapping of thickness and (c) mapping of thickness.

Figure 4 shows the attenuation mapping by ultrasonic testing (transmission mode), in good agreement with the thermal images.

The on-going works aim at relating the thermal diffusivity and ultrasonic attenuation to actual porosity rates.

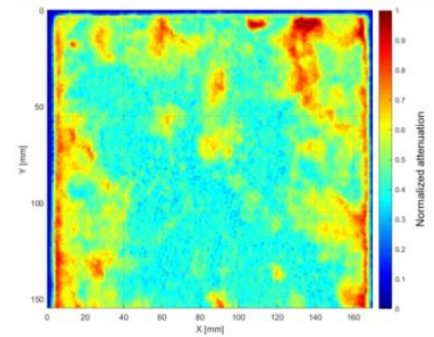

Fig. 4. Normalized attenuation mapping by ultrasonic testing

\section{REFERENCES}

[1] Shepard SM. Advances in pulsed thermography. In: Proceedings of SPIE 4360, Thermosense XXIII, 511, 2001 March 23. http://dx.doi.org/10.1117/12.421032.

[2] Roche, Jean-Michel, and Daniel L. Balageas. "Common tools for quantitative pulse and step-heating thermography-part II: experimental investigation." Quantitative InfraRed Thermography Journal 12.1 (2015): 1 23.

[3] Degiovanni, Alain, and M. Laurent. "Une nouvelle technique d'identification de la diffusivité thermique pour la méthode «flash»." Revue de physique Appliquée 21.3 (1986): 229-237. 